\title{
Influência da espessura de revestimentos de argamassa no desempenho acústico de alvenarias de blocos cerâmicos
}

\author{
Influence of mortar coating thickness on the acoustic \\ performance of ceramic block masonry
}

\begin{tabular}{|c|c|}
\hline & $\begin{array}{l}\text { Sérgio Klippel Filho } \\
\text { Henrique Santos Labres } \\
\text { Fernanda Pacheco } \\
\text { Maria Fernanda de Oliveira } \\
\text { Bernardo Fonseca Tutikian }\end{array}$ \\
\hline $\begin{array}{r}{ }^{3,4} \text { Henrique Santos Labres } \\
\text { 3/tt Performance } \\
\text { São Leopoldo - RS - Brasil } \\
\text { 4Universidade do Vale do Rio dos } \\
\text { Sinos } \\
\text { São Leopoldo - RS - Brasil }\end{array}$ & $\begin{array}{l}\text { Resumo } \\
\text { ercebe-se uma maior preocupação com o desempenho das } \\
\text { edificações habitacionais após a publicação da NBR } 15575 \text { (ABNT, } \\
\text { 2013a), tornando indispensável a avaliação e possível adequação de } \\
\text { sistemas construtivos e seus materiais para atendimento dos } \\
\text { requisitos. Dentre as imposições relativas à habitabilidade, encontra-se o } \\
\text { desempenho acústico, imprescindível na concepção de projetos em ambientes } \\
\text { urbanos, dadas as inúmeras fontes sonoras potentes existentes. O objetivo } \\
\text { deste artigo foi de verificar a influência da espessura de revestimentos de } \\
\text { argamassa estabilizada, aplicados em alvenarias com dois tipos de blocos } \\
\text { cerâmicos, com variação nas espessuras internas e externas de argamassa, } \\
\text { verificando a relação entre o incremento de massa, a curva de isolamento e o } \\
\text { índice de redução sonora ponderado (R } \text { R }_{\mathrm{w}} \text { ). Os ensaios foram executados em } \\
\text { ambiente laboratorial, seguindo as prescrições da ISO 10140-2 } \\
\text { (INTERNATIONAL..., 2010b). Foi possível relacionar a espessura de } \\
\text { revestimento com o desempenho acústico, resultando na redução de insumos, } \\
\text { custos e peso próprio da edificação. Identificou-se que o incremento de } 5 \text { cm } \\
\text { de revestimento de argamassa acarretou em um aumento de até } 7 \text { dB no } \\
\text { isolamento sonoro entre ambientes. }\end{array}$ \\
\hline $\begin{array}{r}\text { 7, } 8 \text { Maria Fernanda de Oliveira } \\
7 \text { Itt Performance } \\
\text { São Leopoldo - RS - Brasil } \\
\text { 8Universidade do Vale do Rio dos } \\
\text { Sinos } \\
\text { São Leopoldo - RS - Brasil }\end{array}$ & $\begin{array}{l}\text { Palavras-chave: Índice de redução sonora. Alvenaria de blocos cerâmicos. Desempenho } \\
\text { acústico. Revestimento de argamassa estabilizada. } \\
\text { Abstract } \\
\text { After the publication of NBR } 15575 \text { (ABNT, 2013a) concern with the performance } \\
\text { of residential buildings has grown markedly, leading companies to evaluate and } \\
\text { adjust construction systems and materials in order to meet the requirements. One } \\
\text { of the requirements is acoustic design, due to the powerful and complex sound } \\
\text { sources. The aim of this study is to analyse the influence of the thickness of } \\
\text { stabilised mortar coatings applied to masonry made with two types of ceramic } \\
\text { blocks, with variation in the internal and external mortar thicknesses, verifying the } \\
\text { relation between mass increase, the insulation curve and the weighted sound } \\
\text { reduction index ( } R_{w} \text { ). The tests were performed in a laboratory environment, } \\
\text { following the requirements of ISO 10140-2 (INTERNATIONAL..., 2010b). The } \\
\text { tests demonstrated that there was a correlation between the thickness of the } \\
\text { coating and the acoustic performance, resulting in the reduction of raw materials, } \\
\text { costs and dead weight of the building. They also showed that an increase of } 5 \text { cm } \\
\text { in the mortar coating resulted in an increase of up to } 7 \text { dB in sound insulation } \\
\text { between rooms. } \\
\text { Keywords: Sound reduction index. Masonry wall systems. Acoustic performance. Stabilized } \\
\text { mortar coating. }\end{array}$ \\
\hline
\end{tabular}

KLIPPEL FILHO, S.; TUTIKIAN, B. F.; OLIVEIRA, M. F.; PACHECO, F.; LABRES, H. S. Influência da espessura de 145 revestimentos de argamassa no desempenho acústico de alvenarias de blocos cerâmicos. Ambiente Construído, Porto Alegre, v. 19, n. 2, p. 145-156, abr./ jun. 2019.

ISSN 1678-8621 Associação Nacional de Tecnologia do Ambiente Construído. http:// dx. doi. org/ 10.1590/ s1678-86212019000200313 


\section{Introdução}

Atualmente considera-se a poluição sonora como um grave problema nos ambientes urbanos, tanto no que diz respeito à saúde dos seres humanos, quanto ao conforto dos usuários na utilização das edificações. Este incômodo é agravado em habitações, visto que se identifica um maior grau de informações presentes no ruído e, desta forma, maior atenção desprendida pelo ouvinte, o que pode impactar na execução de tarefas e lazer, causando desconforto (MASCHKE; NIEMANN, 2007; COWAN, 2016).

Em termos de conforto e desconforto, cabe destacar ainda que, segundo Parmeggiani (2014), os conceitos de desempenho e conforto estão relacionados ao bem-estar dos usuários da habitação, embora não sejam iguais. Enquanto conforto é subjetivo, varia entre as pessoas pela sua percepção, costumes e comportamento, o desempenho consegue ser medido através de ensaios, cálculos, análises de projeto ou outras verificações. A função exercida pela norma de desempenho, a NBR 15575 (ABNT, 2013a), neste cenário, é de transposição de requisitos subjetivos em análises objetivas, qualitativas e quantitativas.

Brito, Soares e Nazari (2013) e Brito (2014) apresentaram estudos focados na identificação das fontes de vibração presentes no meio urbano, afetando a qualidade de vida das pessoas em função do crescimento urbano desordenado e, consequentemente, aproximação das fontes emissoras e receptoras. Tais fontes impactam diretamente na transmissão sonora em edificações, sobretudo nas frequências mais baixas, dificultando o isolamento.

Quando se trata de sistemas de vedações verticais no país, os artefatos produzidos em cerâmica, tais como tijolos e blocos cerâmicos vazados, são largamente utilizados, ressaltando a importância da avaliação do seu desempenho, principalmente com a aplicação de revestimento de argamassa (KAZMIERCZAK, 2010; ASSOCIAÇÃO NACIONAL..., 2016).

De acordo com Fringuellino e Smith (1999) e Hopkins (2007), as perfurações realizadas nos tijolos vazados têm por finalidade aumentar o isolamento térmico e maneabilidade para transporte e construção, assim como, aliviar as cargas na estrutura da edificação e reduzir os custos, quando comparados aos blocos maciços. Em contrapartida, os alvéolos dos blocos acabam diminuindo consideravelmente a massa superficial dos sistemas, amplificando a ocorrência e nível de ressonâncias internas nos blocos. Isto resulta em sistemas construtivos com reduzida capacidade de isolamento sonoro. Neste contexto, Demirkale e Ascigil-Dincer (2017) ainda atribuem aos blocos vazados uma difícil previsão da frequência de ressonância, em função destas características.

Em paralelo, a argamassa é o revestimento mais utilizado nas alvenarias de blocos cerâmicos e apresenta propriedades de endurecimento e aderência compatíveis às necessidades dos sistemas de vedações verticais. Esta age conferindo propriedades adequadas, podendo ser utilizada tanto para revestimento quanto para assentamento dos blocos, agregando características positivas à composição dos sistemas (CARASEK, 2010).

Alternativamente às argamassas produzidas em obra, ou ainda, de origem industrial, comercializadas em sacos, prontas para o uso, a utilização de argamassas estabilizadas, fornecidas por centrais dosadoras, reduz a possibilidade de erro de dosagem, desperdício de materiais, além de aumentar consideravelmente a produtividade no canteiro. O emprego de aditivos estabilizadores de hidratação e incorporadores de ar na composição proporciona trabalhabilidade por períodos de tempos maiores, sem afetar suas propriedades fundamentais no estado endurecido (PANARESE; KOSMATKA; RANDALL, 1991; GUIMARÃES, 2002).

Considera-se, portanto, essencial uma maior atenção por parte dos agentes envolvidos, especialmente dos projetistas, nos aspectos relativos ao desempenho das construções e na concepção e desenvolvimento dos projetos. Ainda, deve-se atentar para a especificação de materiais que concebam sistemas construtivos satisfatórios, cumprindo com os critérios e requisitos legais aplicáveis, bem como atinjam às expectativas dos clientes em casos onde o nível de isolamento elevado é um diferencial (HANSEN, 2005; GUILLEN et al., 2008; OLIVEIRA; MITIDIERI FILHO, 2012; GARG; KUMAR; MAJI, 2013).

De fato, estudos como o de Friedrich, Paixão e Vergara (2010) ratificam a importância do revestimento em paredes de alvenaria, não apenas pelo incremento de massa ao sistema construtivo, mas, também, pela modificação das frequências de ressonância e coincidência, conforme altera-se a espessura da parede. Conforme Fausti et al. (2010), erros diversos oriundos da mão de obra podem resultar em frestas durante processo de assentamento das alvenarias, o que é minimizado com a aplicação das camadas de revestimento. 


\section{Desempenho acústico de edifícios}

Desta forma, de modo a estabelecer níveis mínimos de desempenho em edificações residenciais, a NBR 15575 (ABNT, 2013a), comumente denominada de Norma de Desempenho, estabelece requisitos, dentre eles, o desempenho acústico de sistemas de vedação vertical, horizontal e de instalações, a serem cumpridos independentemente dos sistemas construtivos e materiais adotados. Com isto, é possível fugir dos modelos prescritivos da maioria das normas nacionais em vigor, proporcionando o desenvolvimento de novas tecnologias, mediante cumprimento dos requisitos (SOUZA; KERN; TUTIKIAN, 2018).

Possan e Demoliner (2013) descrevem o desempenho acústico como um dos doze critérios de desempenho baseados na norma ISO 6241 (INTERNATIONAL..., 1984) adaptado e adotado pela norma brasileira de desempenho, visando à satisfação dos usuários. Desempenho este que, de acordo com Santana et al. (2017), diferentemente de países Europeus, e até a publicação da NBR 15575 (ABNT, 2013a, 2013b), aparte a solicitação de medições indicadas pelo Governo Brasileiro, em casos pontuais de saúde pública, ligados, principalmente, à medicina do trabalho, o desempenho acústico foi ignorado no país por muito tempo.

Com relação à acústica de edifícios, os sistemas de vedações verticais e os sistemas de pisos constituem diferentes meios de transmissão do som, com diferentes soluções a serem adotadas para o aumento da perda de transmissão sonora (KLIPPEL FILHO et al., 2016). Nesse contexto, ressalta-se a necessidade de avaliação dos sistemas de vedações verticais frente ao seu isolamento aos sons aéreos. Esta avaliação se faz necessária pela quantidade de fontes sonoras, internas e externas às unidades, necessitando de precaução e prescrição de sistemas construtivos com nível de desempenho adequado e compatível com sua finalidade. Para tanto, os níveis de pressão sonora não devem penetrar em um cômodo ou unidade, em níveis acima da faixa limite indicada (FERREIRA NETO; BERTOLI, 2010).

Juntamente com o cumprimento de requisitos de desempenhos mínimos obrigatórios para cada situação, a redução na transmissão de ruídos para dentro de uma unidade habitacional é pretendida e deve ser fomentada pelas normativas e legislações vigentes. Estas têm por finalidade a segurança e a melhora da qualidade de vida dos usuários, além de proporcionar um diferencial técnico, em níveis de isolamento superiores, com a finalidade de atender às necessidades de clientes que prezam por ambientes com bom isolamento acústico (THOMPSON, 2014).

O isolamento acústico de uma edificação depende da capacidade dos sistemas construtivos empregados em reduzir as transmissões sonoras causadas tanto pelo ruído aéreo quanto pela vibração transmitida estruturalmente. No que diz respeito aos ruídos aéreos, a capacidade do sistema em reduzir a transmissão sonora é diretamente proporcional à massa dos materiais homogêneos que o constituem, sendo que materiais leves possuem menor isolamento, quando comparados com sistemas compostos com os mesmos materiais, porém de maior massa. Todavia, é possível obter maior isolamento acústico em sistemas verticais leves, através da aplicação de um sistema massa-mola-massa que propõe a descontinuidade dos meios e o amortecimento interno das ondas mecânicas (JONES, 2008; SCHIAVONI et al., 2016).

Para tanto, o objetivo deste artigo foi avaliar o índice de redução sonora de sistemas de vedações verticais construídos em alvenarias de blocos cerâmicos estruturais e argamassa estabilizada, verificando a influência da espessura dos revestimentos sobre o desempenho acústico das paredes.

\section{Método}

\section{Definição dos sistemas construtivos}

Para realizar a avaliação do desempenho acústico construíram-se duas paredes de alvenaria de blocos cerâmicos assentados e revestidos em argamassa, em diversas espessuras, aplicadas subsequentemente, observando período de cura e ensaio para cada uma delas. Ambas as paredes foram executadas, simultaneamente, em pórticos móveis de concreto armado (Figura 1) de mesma configuração. Todas as etapas, desde o assentamento dos blocos até a realização dos ensaios, ocorreram dentro do laboratório itt Performance - Unisinos, proporcionando controle de execução e acompanhamento de cura das amostras, em mesmas condições ambientais. 
Figura 1 - Etapas de execução de amostra de alvenaria estrutural: (a) assentamento da alvenaria; (b) chapisco; (c) primeira camada de revestimento; e (d) ensaio

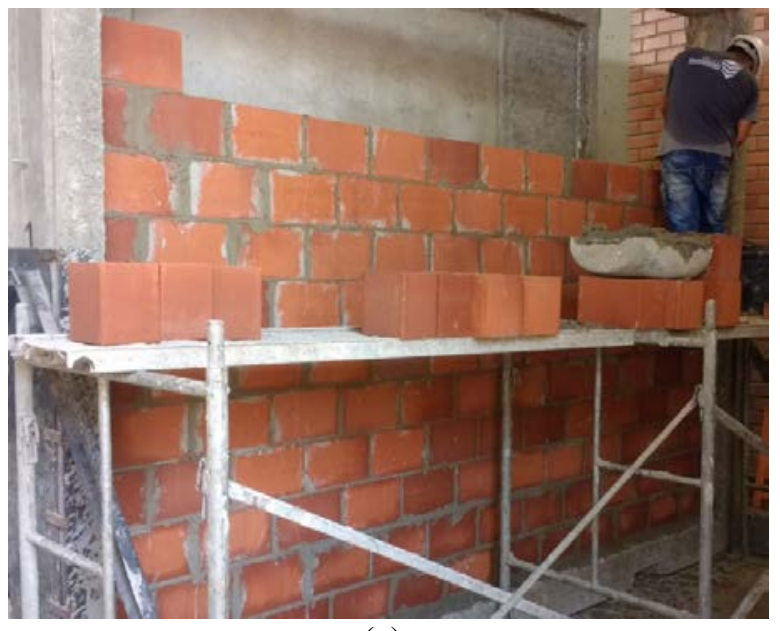

(a)

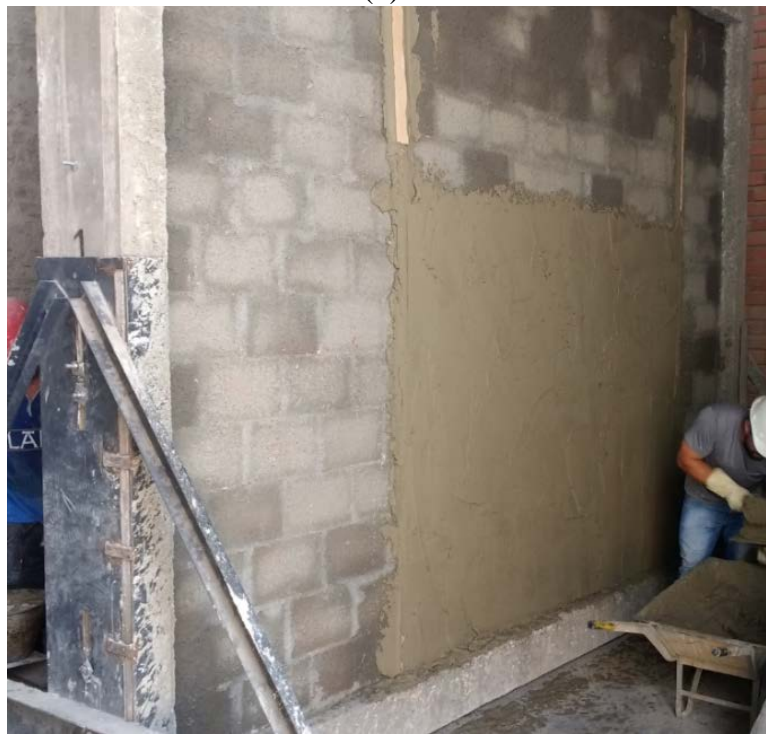

(c)

Aplicaram-se procedimentos executivos e prazos iguais para a construção das paredes, sendo ensaiadas as alvenarias sem revestimento em uma idade de 7 dias. Em seguida, foram retiradas da câmara de ensaio e aplicado chapisco em ambas as faces. Após 3 dias, revestidas com $1 \mathrm{~cm}$ no lado interno e 2 no lado externo, e ensaiadas após novo período de cura de 7 dias. Seguiu-se com a aplicação de mais duas camadas de revestimento, sendo a primeira delas com $1 \mathrm{~cm}$ do lado interno, resultando em $2 \mathrm{~cm}$ nas duas faces. Por fim, após o período de cura e ensaio, a última camada, de $1 \mathrm{~cm}$ no lado externo foi executada, totalizando um revestimento de $2 \mathrm{~cm}$ na face interna e $3 \mathrm{~cm}$ na face externa, ensaiado após 7 dias. Portanto,

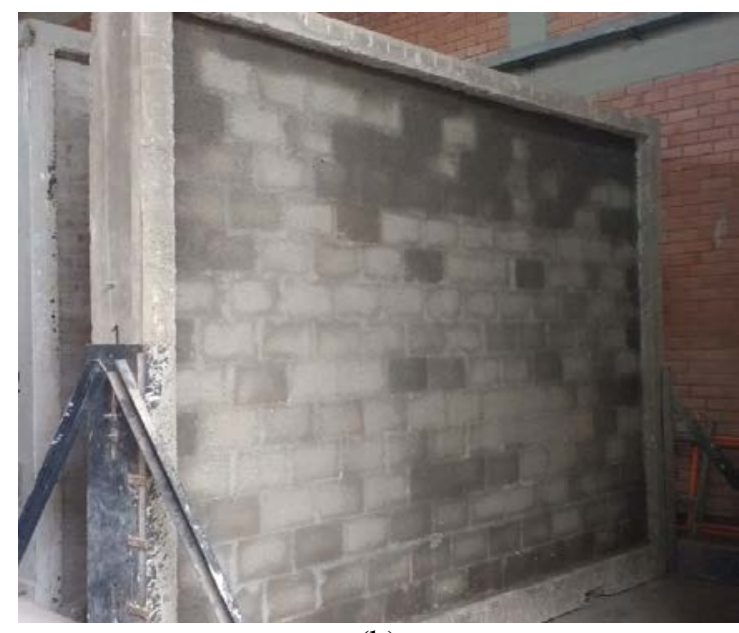

(b)

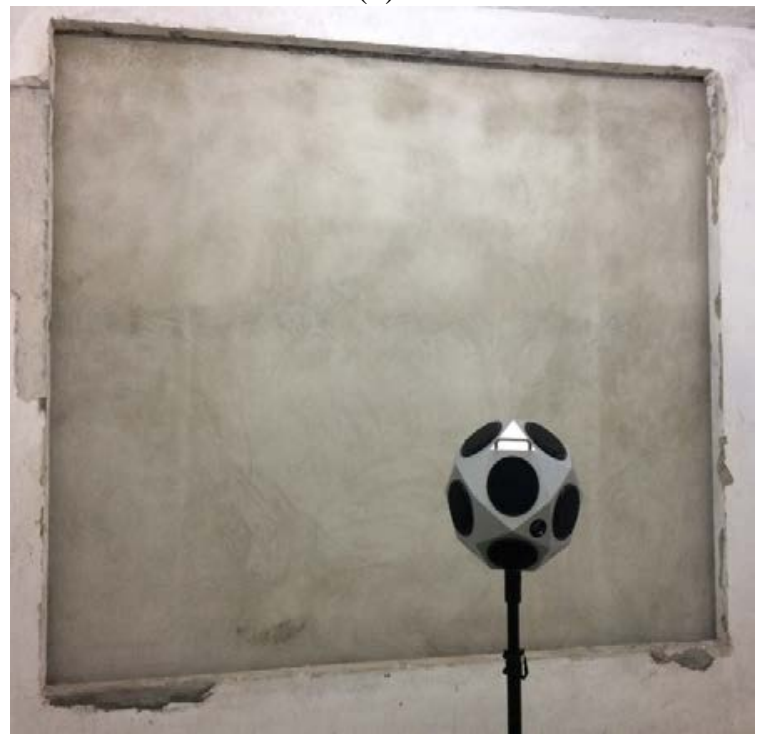

(d)

aprimeira camada de revestimento foi aplicada sobre o chapisco, e as consecutivas aplicadas diretamente à camada anterior de revestimento.

As nomenclaturas, configurações e uma ilustração da geometria de cada bloco, estão presentes na Tabela 1. Para identificação das espessuras de revestimento, foram utilizadas as letras "i” para designar o revestimento na face interna e "e" para o revestimento na face externa, acompanhadas das respectivas espessuras, em centímetro. A determinação de interno e externo é feita para identificar a posição da amostra na câmara de ensaio, onde a face interna é voltada para a câmara receptora e a face externa voltada para a câmara emissora. 
Tabela 1 - Identificação dos sistemas ensaiados: S1 e S2

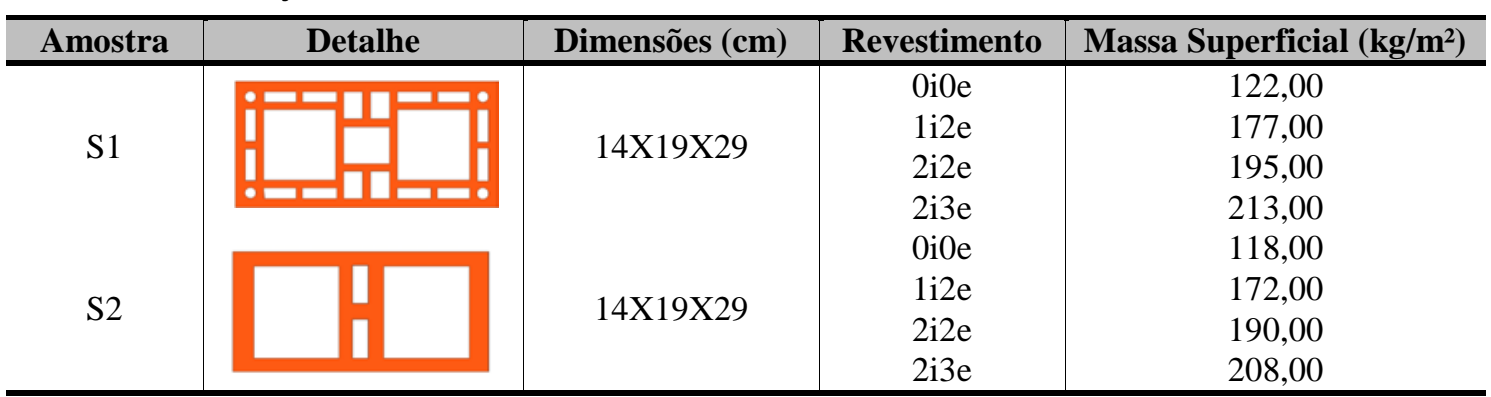

\section{Caracterização dos materiais}

As paredes avaliadas foram compostas de blocos cerâmicos estruturais de mesmo lote e fornecedor, possuindo mesmo processo de fabricação e matéria prima, variando apenas as características geométricas de sua estrutura interna. Estas modificações na configuração da estrutura interna dos blocos somam ao estudo pois visam verificar o comportamento do revestimento em mais de uma situação de rigidez, massa superficial e amortecimento proveniente dos blocos. A argamassa utilizada foi do tipo estabilizada, para assentamento e revestimento das alvenarias, com validade de 36 horas, observando o envio de mesmo lote para cada etapa realizada.

As características físicas dos blocos, ensaiados de acordo com NBR 15270-2 (ABNT, 2017), sendo realizados em 13 amostras selecionadas aleatoriamente de um mesmo lote, frente à Área Bruta $\left(A_{B}\right)$, Área Líquida $\left(A_{\text {liq }}\right)$, massa seca, absorção de água e resistência à compressão axial $\left(f_{b k}\right)$, estão presentes na Tabela 2. Já a argamassa foi ensaiada no seu estado fresco e endurecido por meio de três amostragens, sendo executados metodologias de ensaio específicas, citadas juntamente com os resultados de cada parâmetro, expressos por meio da Tabela 3. O chapisco adotado foi executado com traço 1:3 de cimento CP-IV e areia de granulometria média.

\section{Isolamento aos sons aéreos}

Para determinação do índice de redução sonora (R) realizaram-se ensaios no Laboratório de Acústica do itt Performance - Unisinos, o qual dispõe de duas câmaras reverberantes, sendo a receptora de área igual a $16,52 \mathrm{~m}^{2}$ e volume $57,76 \mathrm{~m}^{2}$; e a emissora, de área igual a $17,45 \mathrm{~m}^{2}$ e volume 61,27 $\mathrm{m}^{2}$ (Figura 2), separadas pela amostra, com área de 10,8 $\mathrm{m}^{2}$, em concordância com a ISO 10140-5 (INTERNATIONAL..., 2010a).
Para todas as avaliações adotaram-se os métodos de precisão, de acordo com as prescrições das normas ISO 10140-2 (INTERNATIONAL..., 2010b) e ISO 10140-4 (INTERNATIONAL..., 2010c), com medições em bandas de terço de oitava, entre as frequências centrais de $100 \mathrm{~Hz}$ e $5000 \mathrm{~Hz}$. As verificações realizadas basearam-se nos valores dos índices de redução sonora por banda de frequência e ponderado, sendo este último, obtido por meio do método de cálculo prescrito pela norma ISO 717-1 (INTERNATIONAL..., 2013). Os equipamentos utilizados nas medições foram todos da marca Brüel and Kjäer, sendo eles: Sound Analyzer Type 2270, Sound Source Omnipower 4292-L, Sound Calibrator Type 4231 e Power Amplifier Type 2734.

\section{Resultados e discussões}

Os valores dos índices de redução sonora do sistema S1 estão expressos na Figura 3, evidenciando a influência das camadas de revestimento na perda de transmissão sonora das alvenarias, especialmente quando comparadas com os sistemas de alvenarias não revestidas.

Observa-se que nas bandas de baixa frequência existe uma maior oscilação nos índices de redução sonora entre as amostras ensaiadas. Isto pode ser explicado pela investigação de Osipov, Mees e Vermeir (1997), os quais atribuem a incerteza ao pouco ou nenhum controle sobre os modos incidentes nas salas, e pelo trabalho de Heissler et $a l$. (2016), evidenciando a ocorrência de valores de incerteza de medição expandida mais elevados ao passo que as bandas de frequência diminuem até o limite inferior da faixa de medição. Percebe-se, ainda, que as frequências de ressonância foram modificadas com a adição das camadas de revestimento ao sistema, com perdas de isolamento acentuadas localizadas entre as bandas de frequências centrais de 125 a $200 \mathrm{~Hz}$. 
Tabela 2 - Características físicas dos dois blocos cerâmicos analisados

\begin{tabular}{c|c|c|c|c|c|c}
\hline Amostra & $\mathbf{A}_{\mathbf{B}}\left(\mathbf{c m}^{2}\right)$ & $\left.\mathbf{A}_{\text {liq }} \mathbf{( c m}^{2}\right)$ & $\mathbf{A}_{\text {liq/A }} \mathbf{A}_{\mathbf{B}}$ & Massa Seca (g) & Absorção de água (\%) & Fbk (MPa) \\
\hline S1 & 406,00 & 165,51 & 0,41 & 5690,20 & 18,49 & 7,00 \\
S2 & 406,00 & 163,35 & 0,40 & 5628,60 & 17,68 & 7,00 \\
\hline
\end{tabular}

Tabela 3 - Características físicas da argamassa estabilizada utilizada

\begin{tabular}{|c|c|c|c|}
\hline \multicolumn{2}{|l|}{ Estado fresco } & \multicolumn{2}{|l|}{ Estado endurecido } \\
\hline $\begin{array}{l}\text { Índice de consistência médio (mm) - } \\
\text { NBR } 13276 \text { (ABNT, 2016) }\end{array}$ & 229,00 & $\begin{array}{l}\text { Resistência à compressão (MPa) - } \\
\text { NBR } 13279 \text { (ABNT, 2005b) }\end{array}$ & 4,28 \\
\hline $\begin{array}{c}\text { Densidade de massa }\left(\mathrm{kg} / \mathrm{m}^{3}\right)-\mathrm{NBR} \\
13278(\mathrm{ABNT}, 2005 \mathrm{a})\end{array}$ & 1834,70 & $\begin{array}{c}\text { Resistência à tração na flexão (MPa) } \\
\text { - NBR } 13279 \text { (ABNT, 2005b) }\end{array}$ & 2,23 \\
\hline $\begin{array}{c}\text { Teor de ar incorporado (\%) - NM } 47 \\
\text { (ABNT, 2002) }\end{array}$ & 3,30 & $\begin{array}{c}\text { Massa específica seca }\left(\mathrm{kg} / \mathrm{m}^{3}\right)-\mathrm{NBR} \\
9778(\mathrm{ABNT}, 2009)\end{array}$ & 1680,00 \\
\hline
\end{tabular}

Figura 2- Sistema de montagem e preparação do ensaio: (a) disposição das câmaras e vão para entrada da amostra; e (b) acoplamento do sistema de vedação

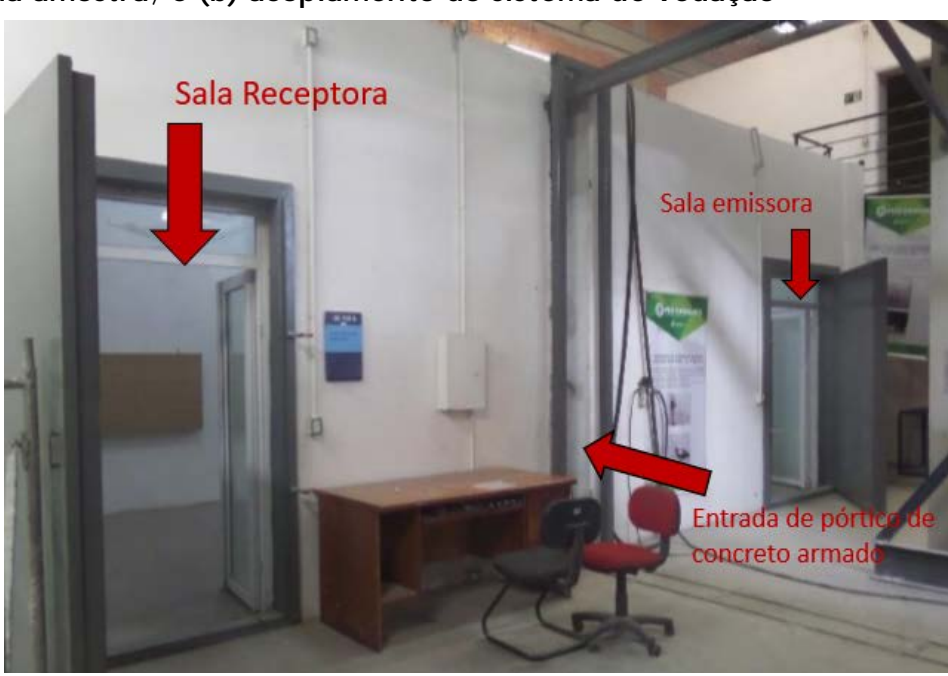

(a)

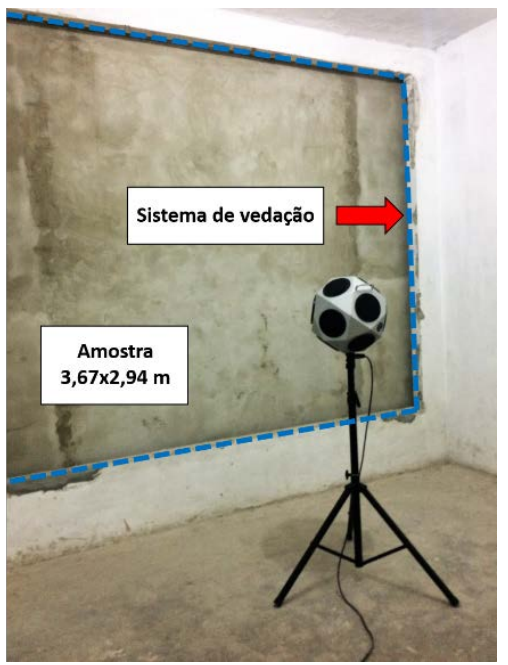

(b)
Nas bandas de média frequência, por sua vez, considerando a capacidade de isolamento acústico do sistema dependente de sua massa superficial, segundo autores como Hopkins (2007), Patrício (2010) e Schiavoni et al. (2016), a perda de transmissão sonora apresentou comportamento crescente até a frequência de coincidência dos sistemas, com exceção da condição de alvenaria não revestida. De fato, a ausência de revestimento aumenta a possibilidade de pequenas frestas entre as juntas, que podem comprometer a estanqueidade do sistema, modificando a curva de isolamento, conforme identificado por Fausti et al. (2010) e Carrascal et al. (2014).

Por conseguinte, a frequência de coincidência foi alterada após a adição das camadas de revestimento, posicionando-se na banda de 2000 $\mathrm{Hz}$, em oposição a queda anterior em 1250 a 1600 Hz. De forma geral, pode-se afirmar que a curva do ensaio da alvenaria sem revestimento tem comportamento bastante distinto em relação à amostra revestida, não podendo ser somente atribuída à influência da massa superficial do sistema. As demais, revestidas de argamassa, demonstram semelhança, especialmente nas bandas de médias e altas frequências, com aumento de isolamento podendo estar atribuído ao acréscimo de massa das camadas de revestimento e vedação de possíveis pontos de fragilidade.

Com relação ao sistema S2, os índices de redução sonora estão expressos na Figura 4, onde, assim como no sistema S1, existe impacto da aplicação do revestimento no índice de redução sonora ao longo das bandas de frequência analisadas, ressaltando as modificações da frequência de ressonância e efeito da coincidência, além dos ganhos advindos do aumento em rigidez e massa do sistema. 
Figura 3 - Comparação entre os índices de redução sonora dos ensaios do sistema S1

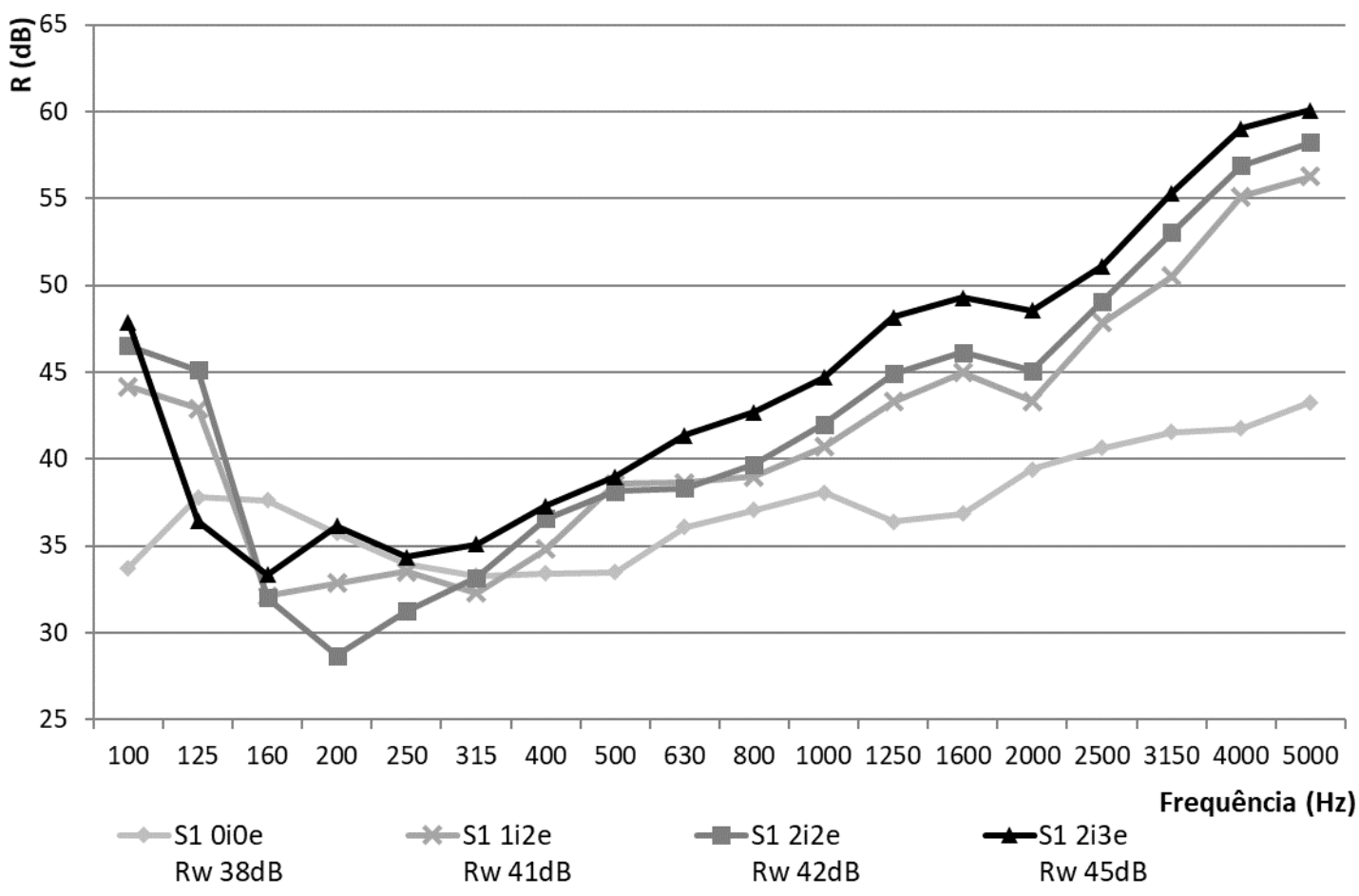

Para esta amostra, conforme supracitado, utilizaram-se as mesmas espessuras, mesmo lote e tipo de material, além dos mesmos métodos construtivos do sistema S1, alterando somente a característica dos blocos cerâmicos. Observa-se, porém, uma menor proporcionalidade entre a adição de massa pelas camadas de argamassa e o ganho de índice de redução sonora, visto que a adição de $4 \mathrm{~cm}$ de argamassa impactou em um aumento de apenas $1 \mathrm{~dB}$ no valor único ponderado, sendo menor, em $3 \mathrm{~dB}$, em relação aos ganhos obtidos no sistema S1. Em bandas de baixa frequência, variações nas frequências de ressonância e oscilações nos índices de redução sonora, tal como no sistema anterior, foram explicadas pela mesma razão supracitada, variando em até $13 \mathrm{~dB}$ entre as configurações de revestimento. O comportamento das curvas de isolamento nas bandas de média frequência também segue os mesmos preceitos, no entanto, a alvenaria não revestida apresentou uma perda de isolamento bastante expressiva entre $1000 \mathrm{~Hz}$ e $2000 \mathrm{~Hz}$, o que não ocorreu nas demais condições com revestimento. Neste caso, a frequência de coincidência da parede revestida encontra-se entre as bandas de $2000 \mathrm{~Hz}$ e $2500 \mathrm{~Hz}$. Assim, ressaltase que, em ambos os sistemas ensaiados, existiu uma maior similaridade entre as curvas de isolamento dos sistemas com revestimento, provavelmente pela minimização de transmissão por falhas e pontos de fragilidade entre as juntas das alvenarias (CARRASCAL et al., 2014; HENDRY; KHALAF, 2001; MATEUS; PEREIRA, 2011). Bies e Hansen (2003) e Patrício (2010) apresentam o comportamento do espectro de elementos construtivos regidos pela lei teórica da massa, considerando a estanqueidade plena dos sistemas, útil na identificação das regiões controladas pela rigidez, massa e amortecimento. Todavia é importante considerar que sistemas heterogêneos podem apresentar variações na curva de isolamento com maior frequência, sendo o seu resultado de difícil previsão.

Na Figura 5 estão presentes de forma gráfica comparações entre os resultados do índice de redução sonora ponderado dos dois sistemas avaliados e uma relação com suas respectivas massas superficiais. 
Figura 4 - Comparação entre os índices de redução sonora dos ensaios do sistema S2

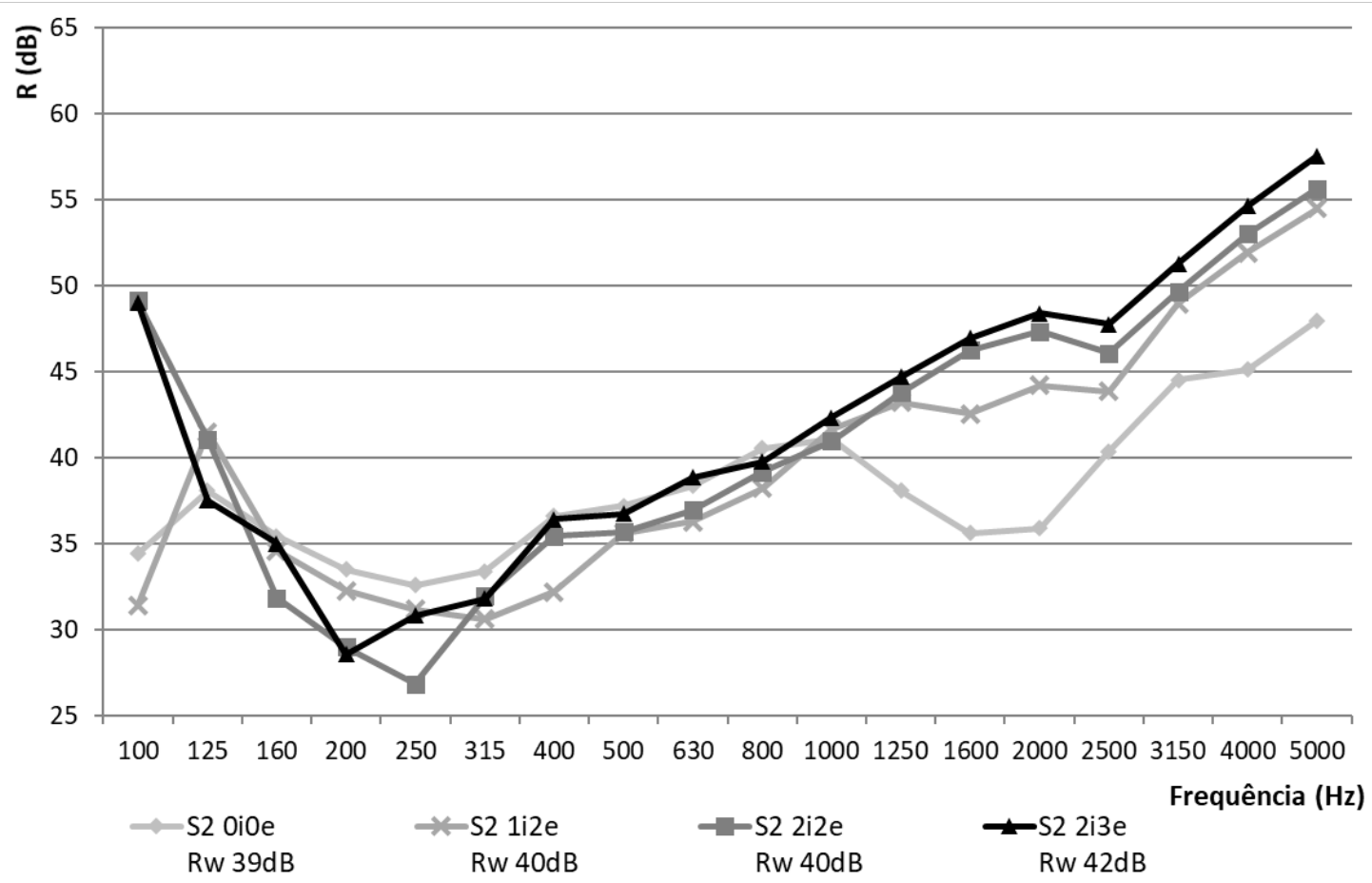

Figura 5- Relação entre o valor único ponderado e a massa superficial dos sistemas S1 e S2

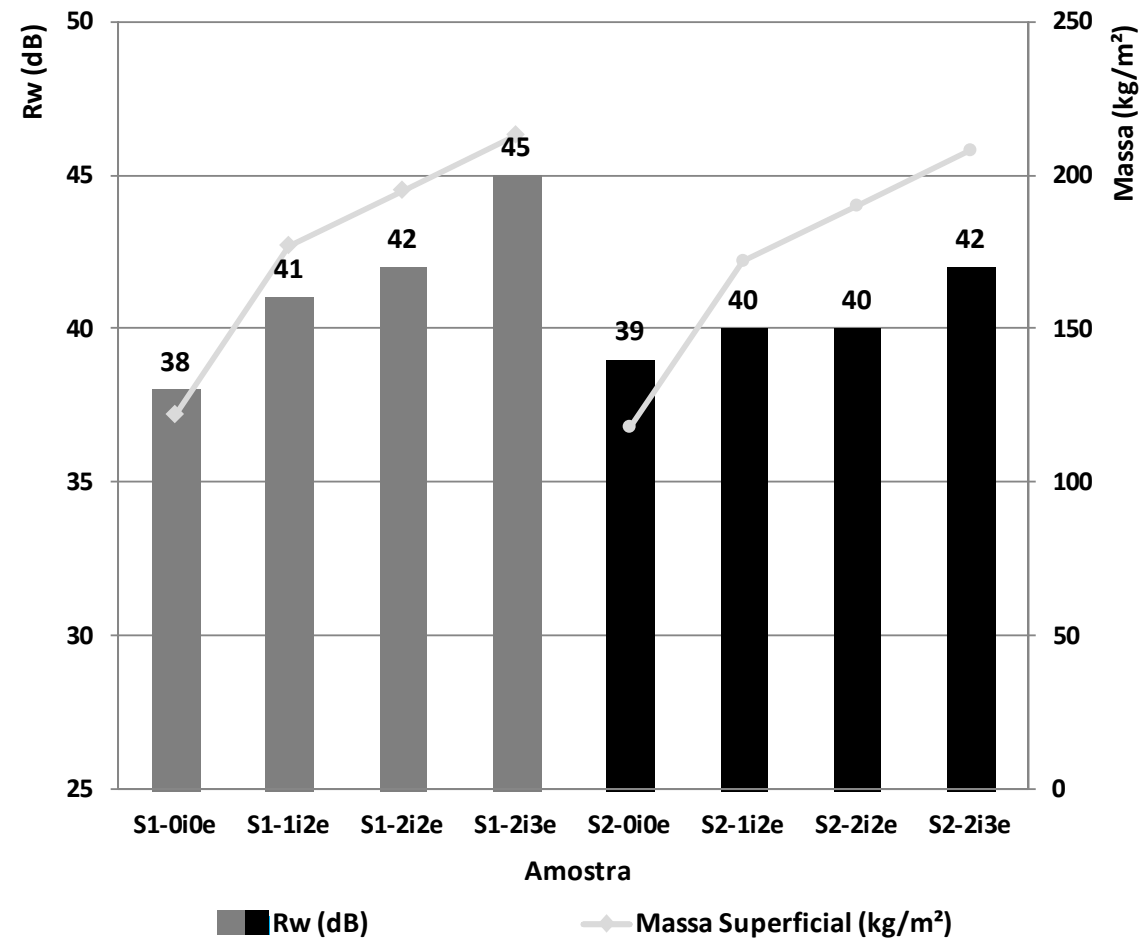

Pode-se identificar a influência positivada aplicação de camadas de revestimento no resultadofinal de cada composição, conforme esperado, tendo em vista a influência considerável do acréscimo de massa superficial em ambos os sistemas. Contudo, estimativas de isolamento em função da massa por unidade de superfície são dificultadas quando os elementos são heterogêneos, conforme discutem alguns autores, como Paixão (2002) e Patrício(2010), podendo

152 Klippel Filho, S.; Labres, H. S.; Pacheco, F.; Oliveira, M. F.; Tutikian, B. F. 
explicar as discrepâncias nos ganhos de isolamento quando comparados os dois sistemas analisados frente às mesmas condições de revestimento. Por meio do gráfico é possível visualizar que a amostra S1 teve um aumento mais escalonado de isolamento em relação ao acréscimo de sua massa superficial, em oposição ao sistema S2.

Ainda, observou-se uma diferença de desempenho entre as duas geometrias de bloco. O bloco do sistema S1 apresentou maiores isolamentos, devido a maior rigidez frente às ondas sonoras, proporcionada por suas paredes internas, como citado no estudo de Fringellino e Smith (1999), onde a rigidez proporcionada pelo layout da estrutura interna influencia no comportamento da redução sonora do sistema. Logo, uma maior rigidez contribuiu para a redução das vibrações transmitidas, fazendo com que o sistema atingisse melhor desempenho, para o caso proposto, com a mesma quantidade de revestimento em argamassa aplicada do sistema S2.

Analisando o sistema S1, pode-se afirmar que, inicialmente, a adição de $55 \mathrm{~kg} / \mathrm{m}^{2}$ de revestimento de argamassa proporcionou um acréscimo de $3 \mathrm{~dB}$ no isolamento do sistema construtivo e, posteriormente, $35 \mathrm{~kg} / \mathrm{m}^{2}$ proporcionou mais $4 \mathrm{~dB}$ de isolamento, transformando o valor único ponderado da alvenaria de $38 \mathrm{~dB}$ para $45 \mathrm{~dB}$.

No sistema S2, o aumento de massa com adição de revestimento de argamassa não foi tão efetivo, podendo representar uma limitação no uso do respectivo bloco cerâmico. O aumento de $72 \mathrm{~kg} / \mathrm{m}^{2}$ de massa, neste caso, representou um aumento de 1 $\mathrm{dB}$, no valor ponderado. Entretanto, a adição de mais $18 \mathrm{~kg} / \mathrm{m}^{2}$, totalizando $90 \mathrm{~kg} / \mathrm{m}^{2}$ em relação a alvenaria sem revestimento, representou um aumento de mais $2 \mathrm{~dB}$, transformando o isolamento acústico do sistema construtivo de 39 $\mathrm{dB}$ para $42 \mathrm{~dB}$.

Percebe-se que, em sistemas heterogêneos, ratificando a discussão de autores como Paixão (2002) e Patrício (2010), o acréscimo de massa não obteve ganhos lineares de isolamento, seja pelas características físicas e geométricas dos materiais, processo executivo ou cura.

\section{Conclusões}

Ambos os sistemas analisados foram compostos por materiais de ampla disponibilidade no mercado brasileiro da construção civil, selecionados para a determinação da influência da espessura de revestimento no isolamento acústico, em dois casos específicos.

A adição de revestimento em argamassa representou ganhos no isolamento de ambos os sistemas, com maior regularidade nas composições da amostra S1, composta por blocos com maior quantidade de vazados. A aplicação de $1,0 \mathrm{~cm}$ de revestimento interno e $2 \mathrm{~cm}$ de revestimento externo nas alvenarias sem revestimento proporcionaram em S1 e S2 ganhos no índice de redução sonora ponderado de $3 \mathrm{~dB}$ e $1 \mathrm{~dB}$, respectivamente. Já na comparação entre amostras já revestidas, na situação $1,0 \mathrm{~cm}$ de revestimento interno e 2,0 cm de revestimento externo para $2 \mathrm{~cm}$ de revestimento interno e 3,0 cm de revestimento externo, em ambos os casos, observou-se ganhos de $4 \mathrm{~dB}$ para S1 e $2 \mathrm{~dB}$ para S2.

As alvenarias sem revestimento obtiveram, inicialmente, índices de redução sonora ponderado de $38 \mathrm{~dB}$ e $39 \mathrm{~dB}$, porém, com a adição de 90 $\mathrm{kg} / \mathrm{m}^{2}$ de revestimento, os incrementos no isolamento foram de $7 \mathrm{~dB}$ e $3 \mathrm{~dB}$, respectivamente, para sistemas S1 e S2. Desta forma, pode-se concluir que os revestimentos em argamassa são mais eficientes para o aumento do isolamento acústico em alvenarias compostas por blocos com maior quantidade de vazados, representando um aumento de, aproximadamente, $1 \mathrm{~dB}$ para cada 1,0 cm de revestimento.

\section{Referências}

\section{ASSOCIAÇÃO BRASILEIRA DE NORMAS}

TÉCNICAS. NBR 13276: argamassa para

assentamento e revestimento de paredes e tetos: determinação do índice de consistência. Rio de Janeiro, 2016.

\section{ASSOCIAÇÃO BRASILEIRA DE NORMAS \\ TÉCNICAS. NBR 13278: argamassa para assentamento e revestimento de paredes e tetos: determinação da densidade de massa e do teor de ar incorporado. Rio de Janeiro, 2005a.}

\section{ASSOCIAÇÃO BRASILEIRA DE NORMAS TÉCNICAS. NBR 13279: argamassa para} assentamento e revestimento de paredes e tetos: determinação da resistência à tração na flexão e à compressão. Rio de Janeiro, 2005b.

\section{ASSOCIAÇÃO BRASILEIRA DE NORMAS \\ TÉCNICAS. NBR 15270: componentes} cerâmicos: blocos e tijolos para alvenaria: parte 2: métodos de ensaios. Rio de Janeiro, 2017.

\section{ASSOCIAÇÃO BRASILEIRA DE NORMAS TÉCNICAS. NBR 15575: edificações} habitacionais: desempenho. Rio de Janeiro, 2013a.

\section{ASSOCIAÇÃO BRASILEIRA DE NORMAS TÉCNICAS. NBR 15575: edificações} habitacionais: desempenho: parte 4: requisitos para os sistemas de vedações verticais internas e externas. Rio de Janeiro, 2013b. 
ASSOCIAÇÃO BRASILEIRA DE NORMAS TÉCNICAS. NBR 9778: argamassa e concreto endurecidos: determinação da absorção de água, índice de vazios e massa específica. Rio de Janeiro, 2009.

ASSOCIAÇÃO BRASILEIRA DE NORMAS TÉCNICAS. NM 47: concreto: determinação do teor de ar em concreto fresco: método pressométrico. Rio de Janeiro, 2002.

ASSOCIAÇÃO NACIONAL DA INDÚSTRIA CERÂMICA. [Setor: dados oficiais]. Disponível em: <www.anicer.com.br>. Acesso em: 13 set. 2016.

BIES, D. A.; HANSEN, C. H. Engineering Noise Control: theory and practice. $3^{\text {rd }}$. ed. London: Spon Press, 2003.

BRITO, L.A; SOARES, A.M.S; NAZARI, B. Vibração: fonte de incômodo à população e de danos as edificações no meio urbano. Ambiente Construído, Porto Alegre, v. 13, n. 1, p. 129-141, jan./mar.2013.

BRITO, L.A. Avaliação das Principais Fontes de Vibração no Meio. Ambiente Construído, Porto Alegre, v. 14, n. 4, p. 233-249, out./dez. 2014.

CARASEK, H. Argamassas. In: ISAIA, G. C. Materiais de Construção Civil e Princípios de Ciências e Engenharia de Materiais. 2. ed. São Paulo:Ibracon, 2010.

CARRASCAL, T.et al. Building Acoustics Throughout Europe. European Cooperation in Science and Technology, v. 1, cap. 10, p. 181212, 2014.

COWAN, J. P. The Effects of Sound on People. London: John Wiley \& Sons, 2016.

DEMIRKALE, S. Y.; ASCIGIL-DINCER, M. Retrofitting Masonry and Cavity Brick Façades For Different Noise Zones Using Laboratory Measurements. Building Acoustics, v.24, n. 2, p.77-100, mar. 2017.

FAUSTI, P. et al. Common Errors During Construction of New Buildings and Effect of Workmanship. In: EUROPEAN SYMPOSIUM, Florence, 2010. Proceedings...Florence, 2010.

FERREIRA NETO, M. D. F.; BERTOLI, S. R. Desempenho Acústico de Paredes de Blocos e Tijolos Cerâmico: uma comparação entre brasil e portugal. Ambiente Construído, Porto Alegre, v. 10, n. 4, p. 169-180,out./dez. 2010.
FRIEDRICH, A. F.; PAIXÃO, D. X.; VERGARA, E. F.; Contribuição do Revestimento no Isolamento Acústico De paredes de Alvenaria. In: ENCONTRO NACIONAL DE TECNOLOGIA DO AMBIENTE CONSTRUÍDO, 13., Canela, 2010.Anais...Canela: ANTAC, 2010.

FRINGUELLINO, M.; SMITH, S. Sound Transmission Through Hollow Brick Walls. Building Acoustics, v. 6, n. 3/4, p. 211-224, sep. 1999.

GARG, N.; KUMAR, A.; MAJI, S. Significance and Implications of Airborne Sound Insulation Criteria in Building Elements For Traffic Noise Abatement. Applied Acoustics, v. 74, n. 12, p. 1429-1435, dec. 2013.

GUILLEN, I.et al. On the Sound Insulation of Masonry Wall Façades. Building and Environment, v. 43, n. 4, p. 523-529, apr. 2008.

GUIMARAES J. E. P. A. Cal: fundamentos e aplicações na engenharia civil.São Paulo. Pini, 2002.

HANSEN, C. Noise Control: from concept to application. London: Taylor \& Francis, 2005.

HEISSLER, R. F. et al.Uncertainty in Measurement Determination in the Sound Reduction Index in Laboratory. In: INTERNATIONAL CONGRESS ON ACOUSTICS, 22.,Buenos Aires, 2016.Proceedings... Buenos Aires, 2016.

HENDRY, A. W.; KHALAF, F. M. Masonry Wall Construction. London: Spon Press, 2001.

HOPKINS, C. Sound Insulation. Oxford: Elsevier, 2007.

INTERNATIONAL ORGANIZATION FOR STANDARDIZATION. ISO 10140: acoustics: laboratory measurement of sound insulation of building elements: part 5: requirements for test facilities and equipment. Geneva, 2010a.

INTERNATIONAL ORGANIZATION FOR STANDARDIZATION. ISO 10140: acoustics: laboratory measurement of sound insulation of building elements: part 2: measurement of airborne sound insulation. Geneva, 2010b.

INTERNATIONAL ORGANIZATION FOR STANDARDIZATION. ISO 10140: acoustics: laboratory measurement of sound insulation of building elements: part 4: measurement procedures and requirements. Geneva, 2010c.

INTERNATIONAL ORGANIZATION FOR STANDARDIZATION. ISO 6241: performance standards in building: principles for their preparation and factors to be considered. Geneva, 1984. 
INTERNATIONAL ORGANIZATION FOR STANDARDIZATION. ISO 717: acoustics: rating of sound insulation in buildings and of building elements: part 1: airborne sound insulation. Geneva, 2013.

JONES, D. Acoustic Treatment For Indoor Areas. In: BALLOU, G. Handbook for Sound Engineers.Burlington: Elsevier, 2008.

KAZMIERCZAK, C. S. Produtos de Cerâmica Vermelha. In: ISAIA, G. C. Materiais de Construção Civil e Princípios de Ciências e Engenharia de Materiais. 2.ed. São Paulo: Ibracon, 2010.

KLIPPEL FILHO, S. et al. Desempenho Acústico em Um Edifício Residencial: classificação superior pela NBR 15575. Acústica e Vibrações, v. 48, p. 93-103, 2016.

MASCHKE, C.; NIEMANN, H. Health Effects of Annoyance Induced by Neighbour Noise. Noise Control Engineering Journal, v. 55, n. 3, p. 348356, may 2007.

MATEUS, D.; PEREIRA, A. Influência de Pequenos Erros de Execução em Obra no Desempenho Acústico de Edifícios: exemplos típicos. TecniAcústica, Cáceres, p.1-8, 2011.

OLIVEIRA, L. A.; MITIDIERI FILHO, C. V. O Projeto de Edifícios Habitacionais Considerando a Norma Brasileira de Desempenho: análise aplicada para as vedações verticais. Gestão\&Tecnologia de Projetos. v. 7, n. 1, p. 90-100, maio 2012.

OSIPOV, A.; MEES, P.; VERMEIR, G. LowFrequency Airborne Sound Transmission Through Single Partitions in Buildings. Applied Acoustics, v. 52, n. 3/4, p. 273-288, apr. 1997.

PAIXÃO, D. X. Caracterização do Isolamento Acústico de Uma Parede de Alvenaria, Utilizando Análise Estatística de Energia (SEA). Florianópolis, 2002. Tese (Doutorado em Engenharia de Produção) - Programa de PósGraduação em Engenharia de Produção, Universidade Federal de Santa Catarina, Florianópolis, 2002.
PANARESE, W.C.; KOSMATKA, S.H.; RANDALL, F.A. Concrete Mansory Handbook for Architects, Engineers, Builders. 5. ed. New York: Portland Cement Association, 1991.

\section{PARMEGGIANI, L. B. Habitabilidade em} edificações segundo a NBR 15575-1: funcionalidade, acessibilidade e conforto antropodinâmico. Trabalho de Conclusão de Curso (Engenharia Civil) - Universidade Federal do Rio Grande do Sul, Porto Alegre, 2014.

PATRÍCIO, J. Acústica nos Edíficios. 6. ed. Lisboa: Verlag Dashöver, 2010.

POSSAN, E.; DEMOLINER. C. A. Desempenho, Durabilidade e Vida Útil das Edificações: Abordagem Geral. Revista Técnico Científica, v. 1, n. 1, p 1-14, out, 2013.

SANTANA, W. B. et al.Rating of Acoustic Performance Levels of NBR 15575 (2013) Based on User Perception: a case study in the Brazilian Amazon. Building Acoustics, v. 24, n. 4, p 239254, nov. 2017.

SCHIAVONI, S. et al.Insulation Materials For the Building Sector: a review and comparative analysis. Renewable \& Sustainable Energy Reviews, v. 62, p. 988-1011, sep. 2016

SOUZA, J. L. P.; KERN, A. P.; TUTIKIAN, B. F. Análise Quantiqualitativa da Norma de Desempenho (NBR no 15.575/2013) e Principais Desafios da Implantação do Nível Superior em Edificação Residencial de Multipavimentos.Gestão e Tecnologia de Projetos, v. 13, n. 1, p 127-144, 2018.

THOMPSON, D. V. Noise Control. In: FAHY, F.; THOMPSON, D. Fundamentals of Sound and Vibration. $2^{\text {nd }}$. ed. Boca Raton: CRC Press, 2014. 


\section{Sérgio Klippel Filho}

Laboratório de Acústica | Itt Performance | Universidade do Vale do Rio dos Sinos | Av. Unisinos, 950, Cristo Rei | CEP 93020-190 | São Leopoldo - RS - Brasil | Tel: (51) 3590-8887 Ramal 3292 | E-mail: engsergio@klippelengenharia.com

\section{Henrique Santos Labres}

Laboratório de Acústica | Itt Performance | Universidade do Vale do Rio dos Sinos | E-mail: hslabres@gmail.com

\section{Fernanda Pacheco}

Analista de Projetos | Itt Performance | Universidade do Vale do Rio dos Sinos | E-mail: fernandapache@unisinos.br

\section{Maria Fernanda de Oliveira}

Coordenadora Laboratório de Acústica | Itt Performance | Universidade do Vale do Rio dos Sinos | E-mail: mariaon@unisinos.br

\section{Bernardo Fonseca Tutikian}

Coordenador itt Performance | Itt Performance | Universidade do Vale do Rio dos Sinos | E-mail: bftutikian@unisinos.br

\section{Revista Ambiente Construído}

Associação Nacional de Tecnologia do Ambiente Construído

Av. Osvaldo Aranha, 99 - 30 andar, Centro

Porto Alegre - RS - Brasil

$$
\text { CEP } 90035-190
$$

Telefone: +55 (51) 3308-4084

Fax: +55 (51) 3308-4054

www. seer. ufrgs. br/ ambienteconstruido

E-mail: ambienteconstruido@ufrgs.br

156 Klippel Filho, S.; Labres, H. S.; Pacheco, F.; Oliveira, M. F.; Tutikian, B. F. 